\title{
Multiscale mechanisms of asphalt performance enhancement by crumbed waste tire rubber: Insight from molecular dynamics simulation
}

Kui Hu ( $\square$ mailhukui@haut.edu.cn )

Henan University of Technology https://orcid.org/0000-0003-0494-8117

Caihua Yu

Henan University of Technology

Yujing Chen

Henan University of Technology

Wei Li

Chang'an University

Chen Guixiang

Henan University of Technology

Wengang Zhang

Shandong University of Technology

\section{Research Article}

Keywords: Molecular dynamic simulation, Crumbed waste tire rubber, Multiscale mechanism, Modified asphalt

Posted Date: March 18th, 2021

DOl: https://doi.org/10.21203/rs.3.rs-319361/v1

License: (c) (1) This work is licensed under a Creative Commons Attribution 4.0 International License.

Read Full License 


\section{Abstract}

The recycling of crumbled waste tire rubber (CWTB) is a major environmental problem facing mankind, and the incorporation of CWTB as a modifier into asphalt is an extremely promising approach. However, the modification mechanism of CWTB to asphalt is not well understood, which restricts the development of CWTB-modified asphalt. In this study, the mechanism of CWTB modification of asphalt was explored in depth by dynamic mechanical analysis (DMA), fluorescence microscopy, scanning electron microscopy (SEM), Fourier transform infrared spectroscopy (FTIR) and molecular dynamics (MD) simulations. The results of the study showed that CWTB enhanced the high temperature performance of the base asphalt. The microscopic mechanism by which this phenomenon occurs is that CWTB has the largest binding energy with the aromatics $(1100-1400 \mathrm{kcal} / \mathrm{mol})$, followed by the saturates $(700-900 \mathrm{kcal} / \mathrm{mol})$, followed by the resins $(200-450 \mathrm{kcal} / \mathrm{mol})$, and the smallest binding energy with the asphaltenes (110$160 \mathrm{kcal} / \mathrm{mol}$ ), which causes CWTB to absorb the light components of the asphalt (aromatics and saturates). In the process of absorbing the light components, CWTB will gradually swell, which causes CWTB to bind more and more tightly with the base asphalt, and eventually the good high temperature performance of CWTB is transferred to the base asphalt. The macroscopic manifestation of this process is that the rutting factor of CWTB modified asphalt is significantly higher than that of virgin asphalt. This study can provide basic theoretical support for the application of CWTB modified asphalt.

\section{Introduction}

The number of cars worldwide has exceeded 1 billion and these vehicles generate more than 2.5 billion scrap tires per year [1,2]. Worryingly, the number of waste tires is still growing at a rate of $2-3 \%$ per year $[3,4]$. Therefore, the recycling of waste tires has attracted worldwide attention. At present, the common means of reuse of waste tires are mainly burning power generation and underground landfill. However, both of these methods have the disadvantages of serious secondary pollution and long metabolic cycles. After years of research and application, adding crumb waste tire rubber (CWTB) to asphalt as a modifier can not only achieve a large consumption of CWTB, but also significantly reduce pavement cracks, which is an ideal way to reuse CWTB $[5,6]$.

Currently, researchers generally agree that the change in the properties of asphalt originates from the interaction between CWTB and the base asphalt [7] and found that there are two phases of CWTB swelling and dissolution in asphalt [8]. Swelling is the phenomenon of volume increase of CWTB in the base asphalt, while dissolution is a phenomenon in which the CWTB is desulfurized and depolymerized at high temperatures $[9,10]$. Both the dissolution and swelling stages have a great influence on the road performance of CWTB modified asphalt. On this basis, the literature $[3,11]$ qualitatively investigated the interaction between various rubbers and asphalt, but still did not come up with a detailed mechanism of CWTB modification of asphalt. Although researchers have conducted many studies on CWTB modified asphalt, the mechanism of CWTB modified asphalt has not been well explained. The main reason is that most of the previous studies used macroscopic experiments to evaluate the effect of CWTB on the macroscopic properties of asphalt, or used various microscopes to make superficial and qualitative 
observations of CWTB modified asphalt, and could not go deeper into the microscopic or molecular scale to get the fundamental mechanism.

Molecular dynamics (MD) simulation is an excellent tool for interpreting material properties from the molecular scale and has attracted strong interest from asphalt researchers [12]. The current application of MD simulations in asphalt is mainly in the areas of interface problems, self-healing problems, and aging problems. The application of MD simulations in interface problems originated from Guo Meng, who pioneered the development and validation of an interface model between asphalt and aggregate, which has profoundly influenced subsequent research [13-15]. The self-healing model usually consists of two asphalt layers with a vacuum zone in between to allow molecular movement [16] and to study the changes in the movement of asphalt molecules under a particular condition [17]. Although MD simulations cannot reproduce the dynamic processes of chemical reactions, the construction of molecular models of asphalt before and after aging allows the evaluation of the effects of aging on the microstate of asphalt $[18,19]$. In addition to these three aspects, there are many fragmented applications of MD simulations [12], and it is believed that MD will solve many old and new problems in the asphalt field in the near future.

The objective of this study was to explore the multi-scale mechanism of CWTB modification of asphalt using MD simulations. CWTB modified asphalt samples were prepared and subjected to dynamic mechanical analysis (DMA). The two-phase structure of CWTB-modified asphalt at different developmental stages was observed by fluorescence microscopy, the microscopic morphology of CWTBmodified asphalt at different developmental stages was observed by scanning electron microscopy (SEM), and the chemical mechanism of CWTB modification of asphalt was analyzed by Fourier transform infrared spectroscopy (FTIR). Finally, natural asphalt was chosen as a representative of CWTB, and the nature of the interaction between CWTB and various molecules of asphalt was explored quantitatively using MD simulations.

\section{Methodology}

\subsection{Construction of CWTB modified asphalt model}

In this study, natural rubber was selected as a representative of CWTB. However, natural rubber is a polymer made of cis-1,4-isoprene as monomer polymerization, and its molecular weight varies with the degree of polymerization, and the computational cost of MD simulation is proportional to the molecular weight of the system $[11,20]$. Therefore, the molecular model of natural rubber needs to be selected. The method used in this study is to calculate the solubility parameter of natural rubber with different polymerization degrees and take the sudden change in the slope of the solubility parameter curve as the model needed for the simulation [21]. The solubility parameter can be calculated by the following equation. 


$$
\begin{aligned}
& \delta=\sqrt{C E D} \\
& C E D=\frac{E_{\text {obl }}}{V}
\end{aligned}
$$

where, $\delta=$ solubility parameter, $C E D=$ cohesion energy density, $E_{c o h}=$ cohesion energy, and $V=$ system volume.

Amorphous cells of natural rubber with different degrees of polymerization were constructed in Materials Studio (MS), they were all geometrically optimized in 500000 steps, and finally the solubility parameters were calculated for different systems. The above processes were performed in the Forcite package of the MS software. The solubility parameters of different natural rubbers are shown in Fig. 1, it can be found that the solubility parameters first increase and then basically equilibrate as the degree of polymerization increases, and the critical point polymerization degree is 12 . Therefore, the natural rubber used in this study was with a polymerization degree of 12 .

The asphalt model was constructed using the assembly method, as it better reflects the complexity of asphalt. The model was used as proposed in the literature [22], which used 12 molecules to represent the four components of asphalt, and the ball-and-stick model of asphalt molecules is shown in Fig. 2. The virgin asphalt sample used in this study was GS-90, and based on the results of the determination of its four-component content (ASTM D4124-09), the number of various asphalt molecules was calculated so that the model four-component content was consistent with the experimental value, and finally the asphalt model could be constructed in the MS software. The number of molecules of the asphalt model in this study, the four-component content of the model and the samples are shown in Fig. 3. It can be found that the various component contents of the asphalt samples are highly consistent with those in the molecular model, which proves the correctness of the asphalt model.

The molecular model of CWTB-modified asphalt was further constructed, and the rationality of the native asphalt model was fully validated in previous studies [21]. The asphalt models used in this study are shown in Fig. 4. It must be mentioned that the content of natural rubber in the CWTB modified asphalt model is $5 \%$. A geometric optimization with a step number of 500000 was performed for each asphalt model with the aim of getting the system to the energy minimum state for subsequent dynamic simulations. The dynamic simulation in this study has two stages, the first stage is 50ps in duration and the system synthesis used is NVT, with the aim of eliminating the high forces present in the system. The second stage is 200ps in duration and the system synthesis used is NPT, which aims to balance the mechanical forces in the system.

In this study, the MD simulation temperature was set to $298.13 \mathrm{~K}$ and the pressure was set to $1 \mathrm{~atm}$, so as to simulate the system variation of modified asphalt at room temperature and pressure. quality was adopted as Ultra-fine, which is to ensure the accuracy of MD simulation. The energy deviation is set to $40,000 \mathrm{kcal} / \mathrm{mol}$ to control the energy change of the simulation. velocities and forces are included for the kinetic simulation trajectory, which is to facilitate our analysis of the various properties of the system. 
During the simulation, Nose was used for Thermostat, Berendsen was used for Barostat, Decay constant was set to $0.1 \mathrm{ps}$, Time step was set to $1 \mathrm{fs}$, Number of steps was set to 200000 , Frame output every was set to 5000 steps, Electrostatic was calculated by Ewald method, and van der Waals was calculated by Atom based method. In addition, COMPASSII force field was used throughout the simulation, which is more accurate compared to COMPASS force field.

The changes of energy, amorphous cell edge length, density and temperature of the CWTB modified asphalt system during the MD simulation are shown in Fig. 5. It can be clearly seen that the energy and temperature of the CWTB modified asphalt system have reached the equilibrium after $15 \mathrm{ps}$. And after $25 \mathrm{ps}$, the system edge length and density also tend to equilibrium, which means that the system has reached the kinetic equilibrium state at this time, and the microscopic quantities of the system can be calculated.

The indicator used in this study to characterize the stability of the interaction is the binding energy, which is currently one of the most commonly used and most effective microscopic characterization quantities. There are many definitions of binding energy, but the most accepted one is the amount of energy or work required to separate two systems. The binding energy can be used to quantify the strength of the bond between two substances. For modified asphalt systems, the state of the bond between the modifier and the asphalt has a direct effect on the performance. The binding energy can be calculated by the following equation.

$$
\begin{aligned}
& E_{\text {boving(AB) }}=E_{\text {bond }}+E_{1 \text { non-bond }} \\
& E_{\text {lverban }}=E_{\text {aाl }}+E_{\text {calanb }}+E_{b c}
\end{aligned}
$$

where $E_{\text {bond }}=$ bonding energy, $E_{\text {Irc }}=$ long range correction, $E_{c o u l o m b}=$ electrostatic energy, $E_{e d W}=\mathrm{vdW}$ energy and $E_{\text {non-bond }}=$ non-bonding energy.

\subsection{Materials and experiment}

The asphalt used in this study was GS-90 provided by Sinopec, whose base properties are shown in Table 1. CWTB of 30 mesh was prepared from waste tires of Henan Huihua Waste Tire Recycling and Treatment Co. Since the CWTB are very hard and have a high melting point, the CWTB cannot be modified to be directly ground into powder and added into the base asphalt. In this study, the CWTB were cracked first, and the amine cracking agent was used, and the rubber powder was made at the end. The various properties of the prepared CWTB are shown in Table 2, and its particle size distribution is shown in Fig. 6. The rubber powder obtained from the cracking was mixed with the base asphalt in a high-speed shear in a certain ratio and sheared at $185^{\circ} \mathrm{C}$ for 3 hours at a speed of $5000 \mathrm{rpm}$, with the aim of allowing the rubber powder to fully interact with the base asphalt to obtain a stable CWTB modified asphalt. 
Table 1. Basic properties of GS-90

\begin{tabular}{ccccc}
\hline \multicolumn{2}{c}{ Project } & GS-90 & After RTOF & Testing standards \\
\hline Penetration & $25^{\circ} \mathrm{C}[0.1 \mathrm{~mm}]$ & 91.0 & 65.5 & ASTM D5 \\
Softening point & TR\&B $\left[{ }^{\circ} \mathrm{C}\right]$ & 48.3 & 91.1 & ASTM D36 \\
Density & $25^{\circ} \mathrm{C}\left[\mathrm{g} / \mathrm{cm}^{3}\right]$ & 1.030 & 1.121 & ASTM D70 \\
Ductility & $15^{\circ} \mathrm{C}[\mathrm{cm}]$ & 105.2 & 76.2 & ASTM D113 \\
\hline
\end{tabular}

Table 2. Basic properties of CWTB

\begin{tabular}{ccccccc}
\hline Water [\%] & Rubber hydrocarbon [\%] & Ash [\%] & Acetone extracts [\%] & Natural rubber [\%] & Density [g/cm ${ }^{3}$ ] & Carbon black [\%] \\
\hline 0.7 & 49 & 3 & 4 & 37 & 1.20 & 6
\end{tabular}

In this study, FTIR tests, SEM tests, fluorescence microscopy tests and DMA were performed successively on virgin and CWTB modified asphalts with the aim of exploring the multi-scale modification mechanism of CWTB modified asphalt in combination with MD simulations. The SEM uses the TM3030PLUS ${ }^{\mathrm{TM}}$. FTIR by Bruker Spectroscopy, Germany, model VERTEX $70^{\mathrm{TM}}$. The model number of fluorescence microscope is BSM-600E ${ }^{\mathrm{TM}}$, the optical magnification is $40 \mathrm{X}-2000 \mathrm{X}$, provided by Shanghai BIMU Instruments Co. And the DSR model is Bohlin Gemini ${ }^{\mathrm{TM}}$ by Xi'an Minx Testing Equipment Co., Ltd. and the test standard is AASHTO T 315. The technical route of this study is shown in Fig. 6.

\section{Results And Discussion}

\subsection{Rutting resistance of CWTB modified asphalt and virgin asphalt}

The rutting factors of CWTB modified asphalt and base asphalt are shown in Fig. 8. The rutting factor of both base asphalt and CWTB modified asphalt decreases significantly with the increase of temperature. This phenomenon is a fundamental property of asphalt materials, also known as temperature sensitivity, and is responsible for the occurrence of high temperature rutting distress.

The rutting factor of the CWTB modified asphalt is greater than that of the base asphalt regardless of the temperature, which indicates that CWTB have an enhanced effect on the high temperature performance of the asphalt. This is because CWTB absorbs some of the aromatics and saturates in the base asphalt, causing the relative asphaltenes and resins content of the base asphalt to rise. At the same time, CWTB itself has good high temperature performance, so the rutting factor of CWTB modified asphalt is higher than that of the base asphalt. 


\subsection{Two-phase structural state of CWTB modified asphalt}

The two-phase structures of CWTB modified asphalt at different development times are shown in Fig. 9. It can be found that the fluorescence microscope picture shows a clear two-phase structure, where the black phase is the CWTB phase and the red phase is the asphalt phase. This indicates that CWTB is incompatible with the base asphalt and that CWTB and asphalt are easily separated.

In addition, the area of CWTB phase in the modified asphalt increases significantly and the volume of waste tire floc becomes larger as the development time increases. This supports the conclusion that CWTB absorbs light components, and the reason for the larger CWTB phase with longer development time is that more lighter components are adsorbed by CWTB as the development time grows, which often predicts better road performance.

\subsection{Micro-interface morphology of CWTB modified asphalt}

The SEM morphology of CWTB modified asphalt in different development periods is shown in Fig. 10. It can be found that CWTB is not dissolved in the base asphalt, which indicates that CWTB is not completely compatible with the base asphalt, and this result is consistent with the fluorescence microscopy observation. However, the boundary between CWTB and asphalt is not clear, which indicates that CWTB will interact with the base asphalt.

As the development time increased, the volume of CWTB increased significantly, which is the result of the association of CWTB with the lighter part of the asphalt. In addition, it has to be mentioned that the surface of CWTB has become smoother when the development time is $4 \mathrm{~h}$, which will be the compatibility of CWTB with the base asphalt. This phenomenon predicts that the development time of CWTB should not be too long, because it will increase the tendency of phase separation of CWTB modified asphalt.

\subsection{Effect of waste tires on functional groups of base asphalt}

The FTIR results of the base asphalt and CWTB modified asphalt are shown in Fig. 11. It can be clearly found that the fluctuations of FTIR curves of base asphalt and CWTB modified asphalt are basically the same, which indicates that the functional groups of the two asphalt systems are basically the same. In other words, there is no chemical reaction between the waste tire and the base asphalt, and CWTB is mainly a physical modification of the base asphalt.

The FTIR curves of both the base asphalt and the CWTB modified asphalt have distinct peaks at wavenumbers $1300-1500 \mathrm{~cm}^{-1}$ and $2800-3000 \mathrm{~cm}^{-1}$. The functional groups corresponding to this wave number are saturated hydrocarbon groups $(\mathrm{CH})$ and methylene $\left(\mathrm{CH}_{2}\right)$, respectively. Observation of the wave intensity reveals that the peak of the base asphalt is between $0.08-0.14 \%$, while the CWTB modified asphalt is less than $0.06 \%$. The reason why the peak of the CWTB modified asphalt is lower than that of 
the base asphalt is that the CWTB modified asphalt will join and crosslink with the light components, which causes a significant reduction in the $\mathrm{CH}$ and $\mathrm{CH}_{2}$ functional groups.

\subsection{Stability of CWTB interaction with asphalt}

The binding energy and its composition between CWTB and various molecules of asphalt are shown in Fig. 12. The binding energy indicates the stability of the interaction, that is, the order of the magnitude of the stability of the interaction between CWTB and the molecules of the asphalt system is as follows: NAPHPN $>$ NADOCHN $>$ SHopane $>$ Ssqualane $>$ PATIRE $>$ PATMBO $>$ PAPNH $>$ PAQNP $>$ AThiophene $>$ APyrrole $>$ APhenol $>$ PABBTP. Overall, CWTB interacts most stably with aromatics, with binding energies between them ranging from $1100-1400 \mathrm{kcal} / \mathrm{mol}$. CWTB interacts secondarily with saturates, with binding energies between them ranging from $700-900 \mathrm{kcal} / \mathrm{mol}$. CWTB interacts less stably with resins than saturates, with binding energies between them ranging from 200-450 kcal/mol. The interaction between CWTB and asphaltenes is the most unstable, with a binding energy of $110-160 \mathrm{kcal} / \mathrm{mol}$. The binding energy data indicate that CWTB can easily associate with light components and that rubber absorbs lighter components in asphalt. The results of the binding energy fundamentally reveal the nature of the interaction between CWTB and asphalt, and also reveal the mechanism of the formation of the biphasic structure of CWTB-modified asphalt.

From the composition of the binding energy, the bond energy between CWTB and all asphalt molecules is $0 \mathrm{kcal} / \mathrm{mol}$, which indicates that the interaction between CWTB and asphalt is physical. This

explains the FTIR results. The value of vdW energy is much larger than that of electrostatic energy relative to electrostatic energy. In other words, the main type of intermolecular interaction in the CWTB modified asphalt system is the vdW force, not the electrostatic force.

\section{Conclusion}

The above conclusions were obtained by exploring the multi-scale mechanism of CWTB modification of asphalt using MD simulation, FTIR, SEM, fluorescence microscopy and DMA.

1. the optimum degree of polymerization of natural rubber in MD simulations was 12 .

2. CWTB will interact with the base asphalt and the interaction is enhanced with the development time, but the surface of CWTB will become smooth, which will increase the tendency of CWTB separation from asphalt.

3. The modification mechanism of CWTB to asphalt is that CWTB has the strongest interaction with aromatics, followed by saturates, resins third, and the weakest interaction with asphaltenes, which causes CWTB to absorb part of saturates and aromatics and swell. However, CWTB does not have peanut chemical reaction with asphalt, and the modified asphalt system shows an obvious twophase structure due to the incompatibility between CWTB and the base asphalt. With the growth of development time, the interface between CWTB and base asphalt becomes more and more blurred, 
and various properties of CWTB are gradually transferred to the base asphalt, causing the growth of asphalt rutting factor.

\section{Declarations}

\section{Acknowledgments}

This work was financially supported by Innovative Funds Plan of Henan University of Technology (No. 2020ZKCJ05), Science and Technology Planning Project of Henan Province of China (No.

192102310229), the Cultivation Plan for Youth Backbone Teachers of Institution of Higher Education by Henan Province (No. 2019GGJS086), the Cultivation Plan for Youth Backbone Teachers by Henan University of Technology. Key Science and Technology Research project of Henan provincial department of education (No. 21A580002) and the foundation for Distinguished Young Talents of Henan University of Technology (No. 2018QNJH09).

\section{Author contribution}

Kui Hu: Conceptualization, Investigation, Methodology, Writing - original draft. Caihua Yu: Formal analysis, Software, Methodology, Writing - review \& editing. Yujing Chen: Conceptualization, Writing review \& editing; Wei Li: Validation, Resources. Guixiang Chen: Supervision, Conceptualization, Investigation, Validation, Funding acquisition. Wengang Zhang: Supervision, Conceptualization, Writing review \& editing.

\section{Author contribution}

All data generated or analyzed during this study are included in this published article.

\section{Declarations}

Ethics approval and consent to participate: The manuscript is prepared in compliance with the Ethics in Publishing Policy as described in the Guide for Authors. The manuscript is approved by all authors for publication.

Consent for publication: The consent for publication was obtained from all participants.

Conflict of interest: The authors declare that they have no conflict of interest.

\section{References}

1. Long Z, Zhou S, Jiang S, Ma W, Ding Y, You L, Tang X, Xu F (2021) Revealing compatibility mechanism of nanosilica in asphalt through molecular dynamics simulation. J Mol Model 27:1-14 
2. Behnood A, Gharehveran M (2019) Morphology, rheology, and physical properties of polymermodified asphalt binders. Eur Polym J 112:766-791

3. Guo F, Zhang J, Pei J, Zhou B, Hu Z (2019) Study on the Mechanical Properties of Rubber Asphalt by Molecular Dynamics Simulation. J Mol Model 25:1-8

4. Cong P, Guo X, Mei L (2020) Investigation on rejuvenation methods of aged SBS modified asphalt binder. Fuel 279:118556

5. Rz $z^{2}$ ek L, Turk M, Tušar M (2020) Increasing the rate of reclaimed asphalt in asphalt mixture by using alternative rejuvenator produced by tire pyrolysis. Constr Build Mater 232:117177

6. Landi D, Marconi M, Bocci E, Germani M (2020) Comparative life cycle assessment of standard, cellulose-reinforced and end of life tires fiber-reinforced hot mix asphalt mixtures. J Clean Prod 248:119295

7. Dong Z, Zhou T, Luan H, Williams R, Wang P, Leng Z (2019) Composite modification mechanism of blended bio-asphalt combining styrene-butadiene-styrene with crumb rubber: A sustainable and environmental-friendly solution for wastes. J Clean Prod 214:593-605

8. Cao R, Leng Z, Yu H, Hsu S (2019) Comparative life cycle assessment of warm mix technologies in asphalt rubber pavements with uncertainty analysis. Resour Conserv Recy 147:137-144

9. Wang S, Gao Y, Yan K, You L, Jia Y, Dai X, Chen M, Diab A (2020) Effect of long-term aging on waste tire rubber and amorphous poly alpha olefin compound modified asphalt binder and its mixtures. Constr Build Mater 272:1-13

10. Wang M, Li R, Wen Y, Pei J, Xing X, Chen Z (2019) Rheological and aging behaviors of liquid rubber modified asphalt binders. Constr Build Mater 227:1-9

11. Guo F, Zhang J, Pei J, Zhou B, Falchetto A, Hu Z (2020) Investigating the interaction behavior between asphalt binder and rubber in rubber asphalt by molecular dynamics simulation. Constr Build Mater 252:1-10

12. Chen Z, Pei J, Li R, Xiao F (2018) Performance characteristics of asphalt materials based on molecular dynamics simulation - A review. Constr Build Mater 189:695-710

13. Guo M, Tan Y, Wei J (2018) Using Molecular Dynamics Simulation to Study Concentration Distribution of Asphalt Binder on Aggregate Surface. J Mater Civil Eng 30:04018075

14. Luo D, Guo M, Tan Y (2018) Molecular Simulation of Minerals-Asphalt Interfacial Interaction. Minerals 8:1-10

15. Guo M, Tan Y, Wang L, Hou Y (2017) Diffusion of asphaltene, resin, aromatic and saturate components of asphalt on mineral aggregates surface: molecular dynamics simulation. Road Mater Pavement 18:149-158

16. Xu G, Wang H (2017) Molecular dynamics study of oxidative aging effect on asphalt binder properties. Fuel, 2017. 188: 1-10

17. Sun D, Lin T, Zhu X, Tian Y, Liu F (2016) Indices for self-healing performance assessments based on molecular dynamics simulation of asphalt binders. Comp Mater Sci 114:86-93 
18. Chen Z, Yi J, Zhao H, Luan H, Xu M, Zhang L, Feng D (2020) Strength development and deterioration mechanisms of foamed asphalt cold recycled mixture based on MD simulation. Constr Build Mater $1-16$

19. Long Z, You L, Tang X, Ma W, Ding Y, Xu F (2020) Analysis of interfacial adhesion properties of nanosilica modified asphalt mixtures using molecular dynamics simulation. Constr Build Mater 255:119354

20. Yu H, Leng Z, Zhou Z, Shih K, Xiao F, Gao Z (2017) Optimization of preparation procedure of liquid warm mix additive modified asphalt rubber. $J$ Clean Prod 141:336-345

21. Feng Y, Yu C, Hu K, Chen Y, Liu Y, Zhang T, A study of the microscopic interaction mechanism of styrene-butadiene-styrene modified asphalt based on density functional theory. Mol Sim 1-12

22. Li D, Greenfield M (2014) Chemical compositions of improved model asphalt systems for molecular simulations. Fuel 115:347-356

\section{Figures}

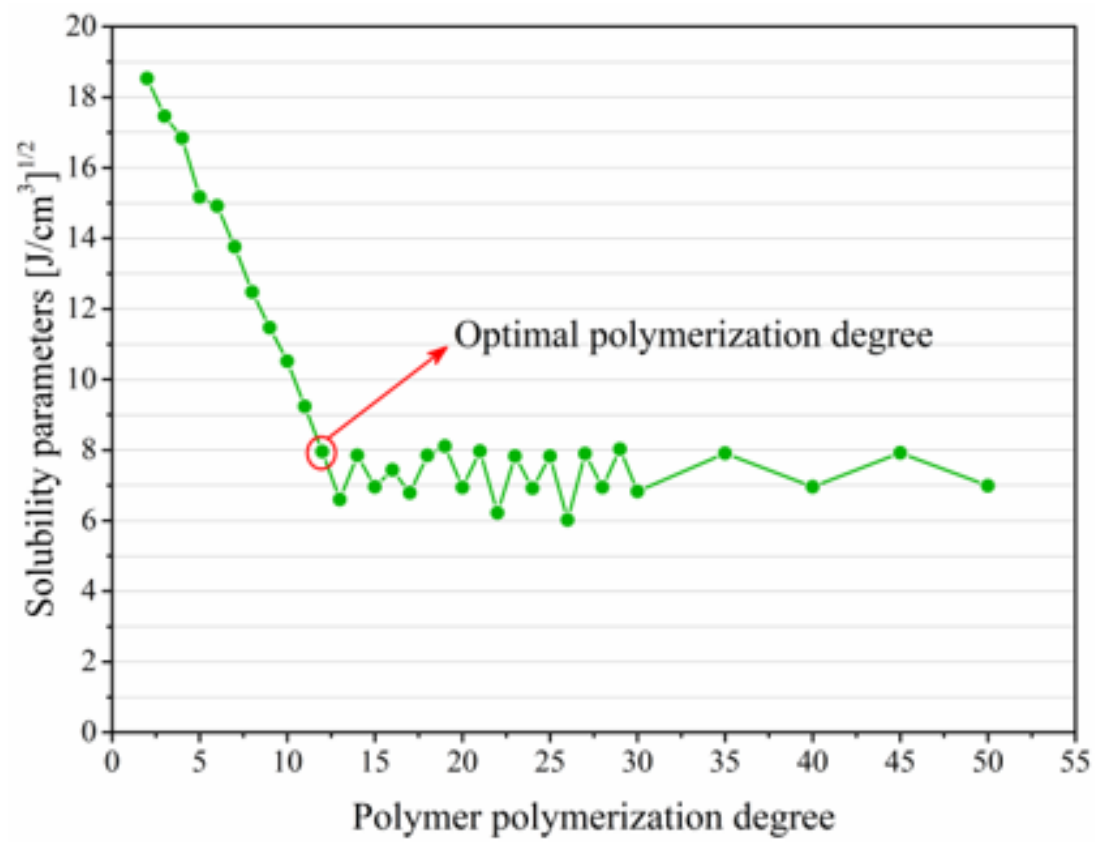

\section{Figure 1}

Solubility parameters of different natural rubbers 

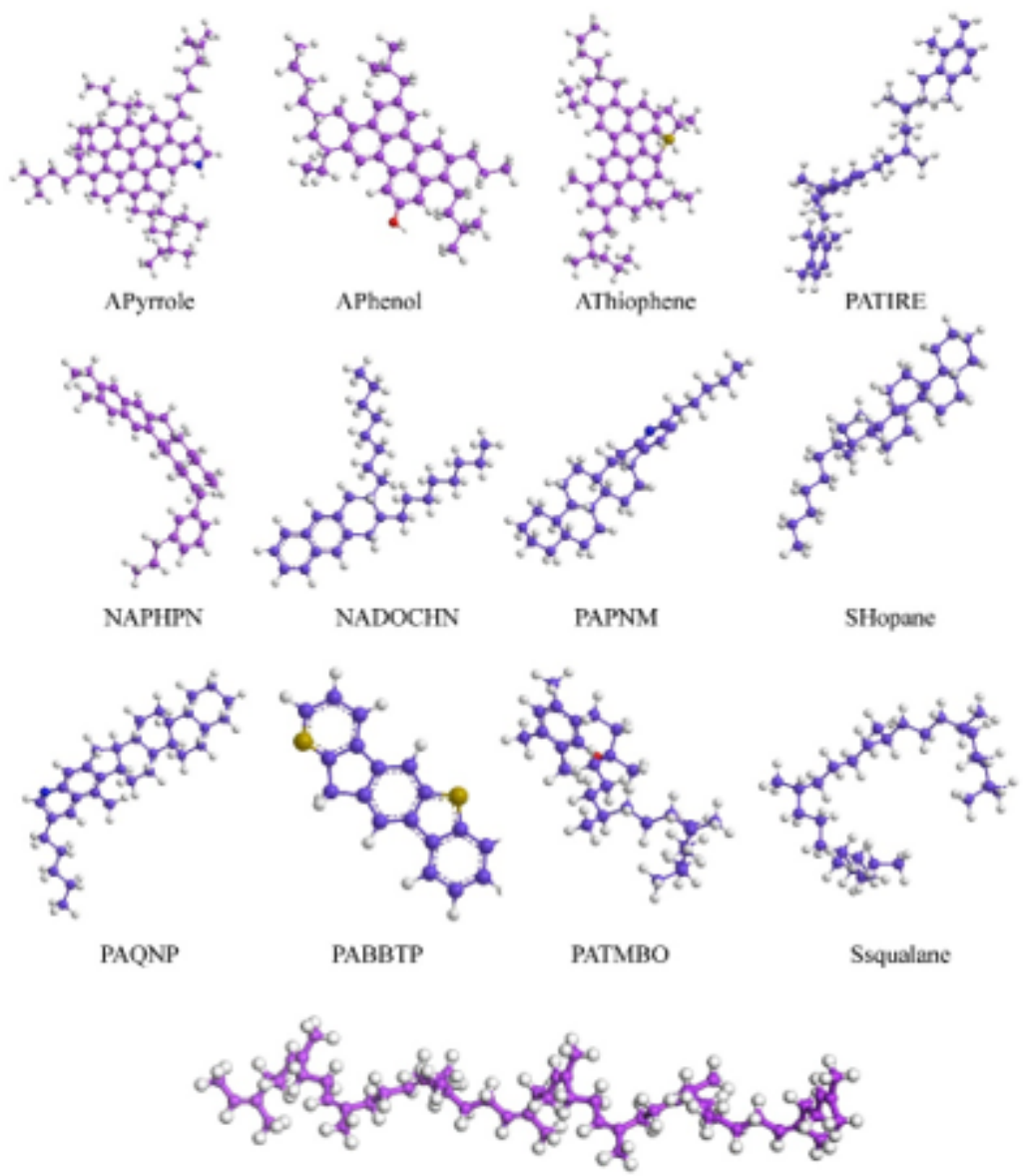

Natural Rubber

Figure 2

The molecular model of asphalt used in this study

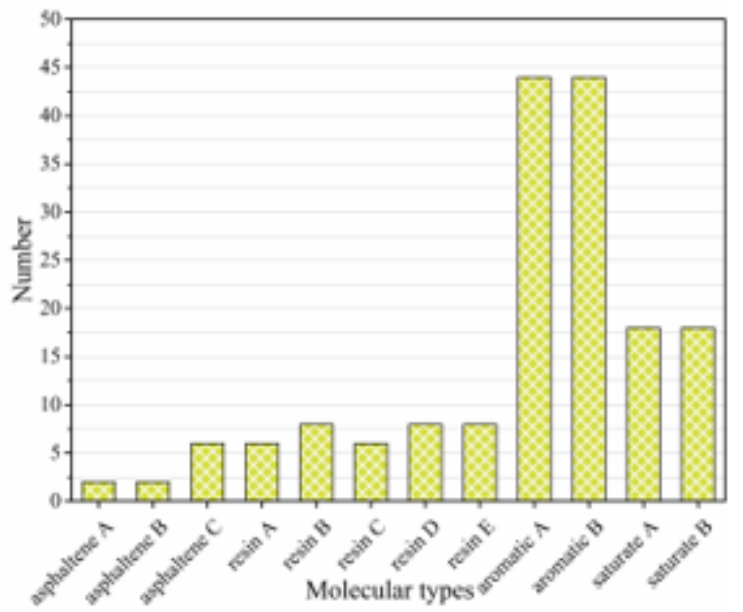

(a)

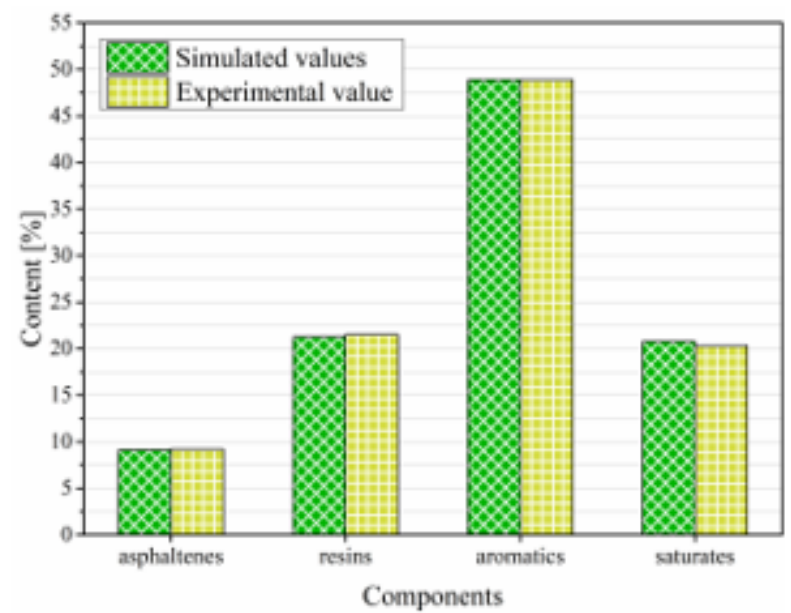

(b)

Figure 3 
The number of various molecules in the asphalt model (a) and the four components of the model and sample

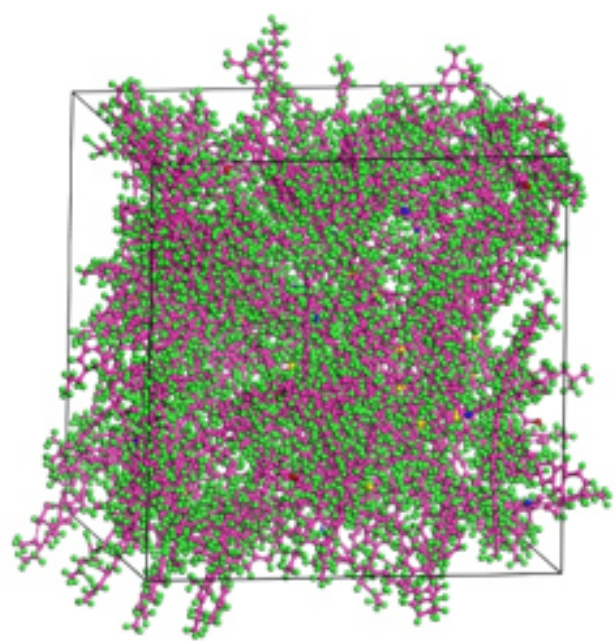

(a)

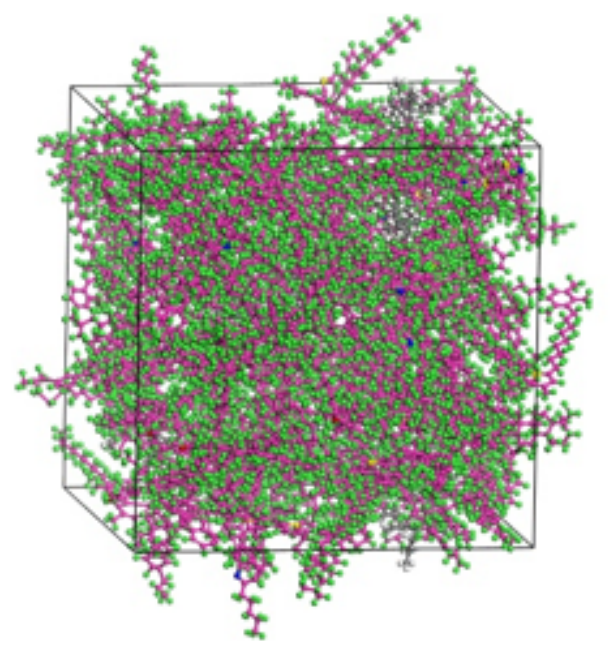

(b)

\section{Figure 4}

The number of various molecules in the asphalt model (a) and the four components of the model and sample 


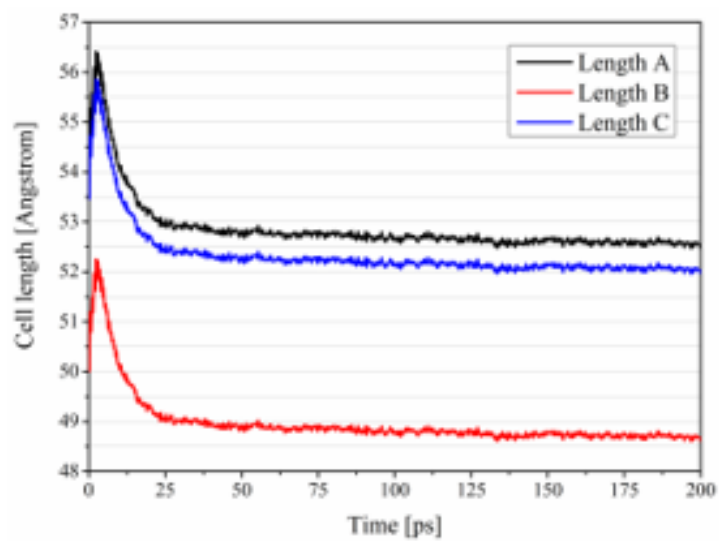

(a)

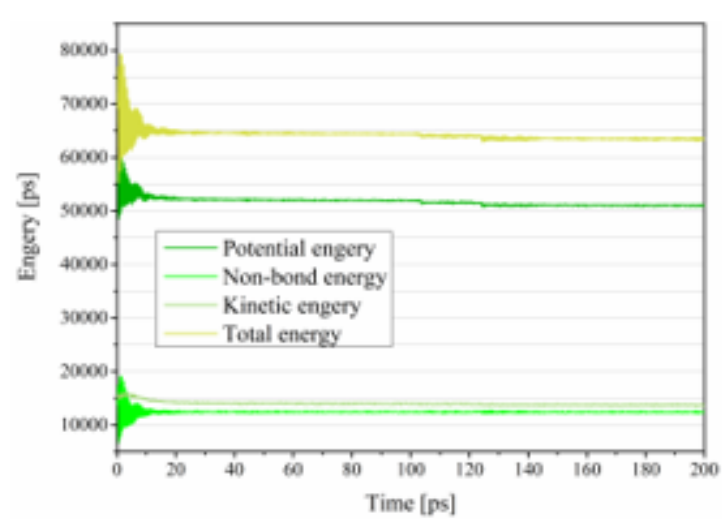

(c)

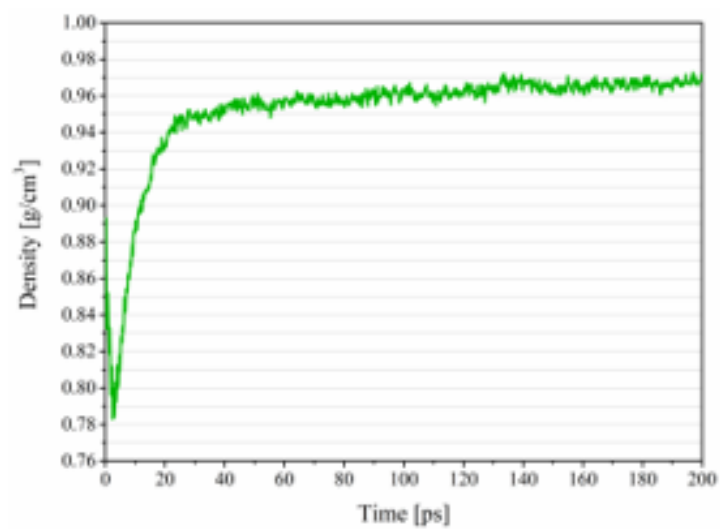

(b)

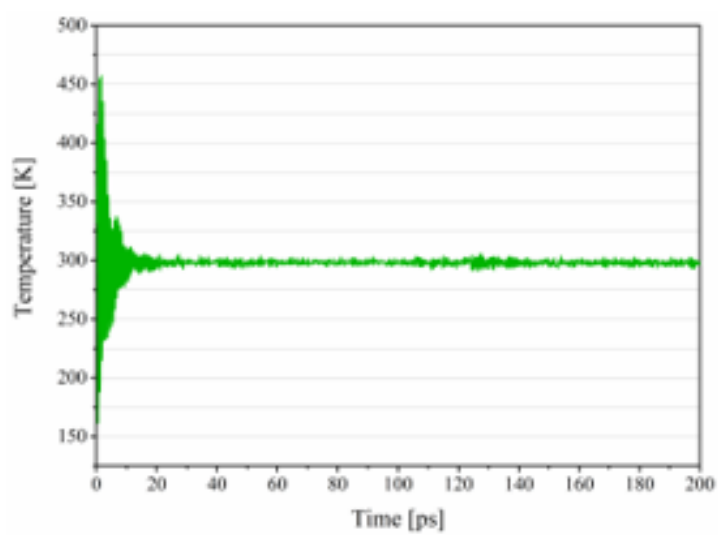

(d)

\section{Figure 5}

Trends in cell length (a), density (b), energy (c) and temperature (d) fluctuations during MD simulations 


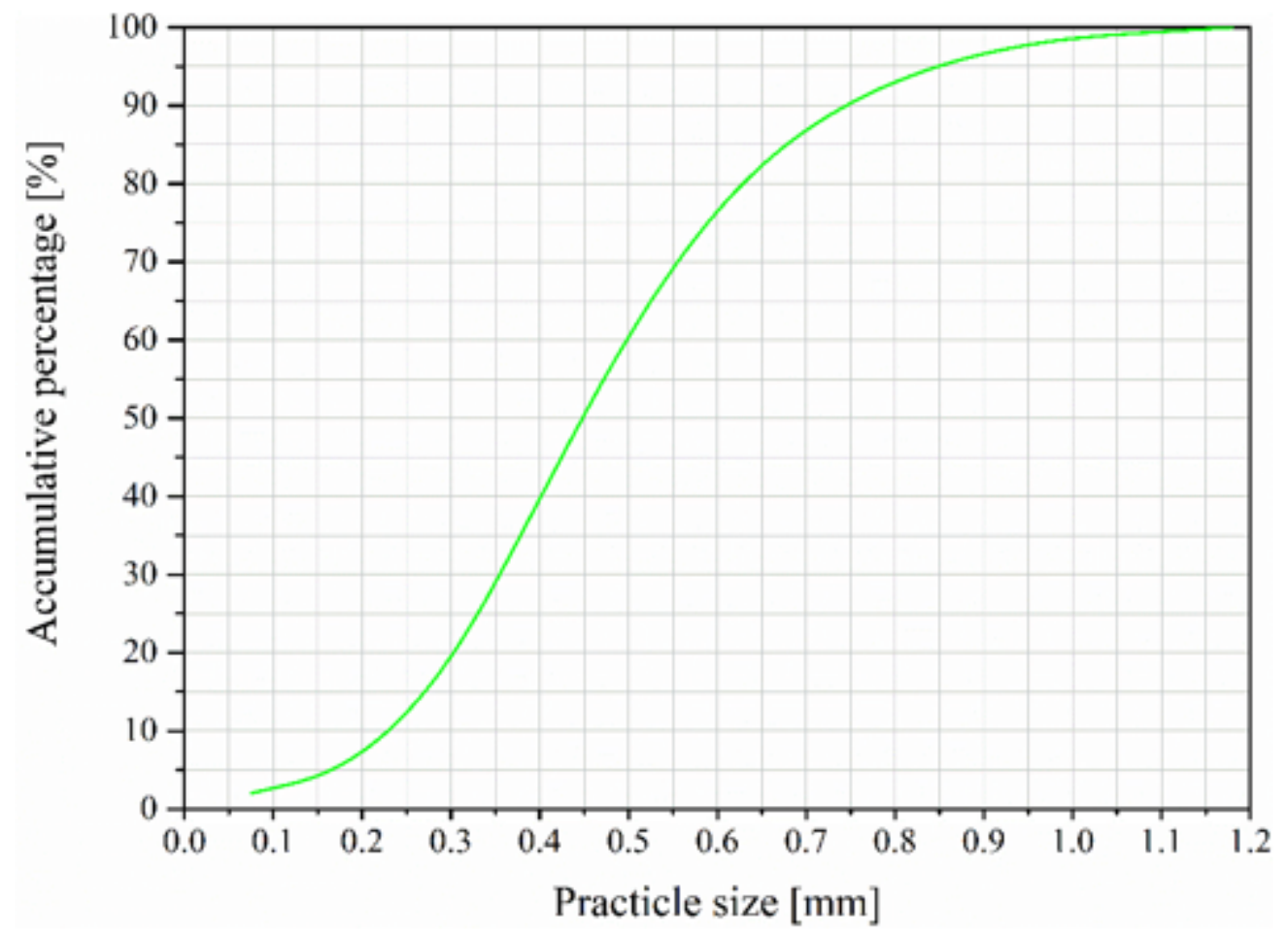

Figure 6

Particle size distribution of CWTB 


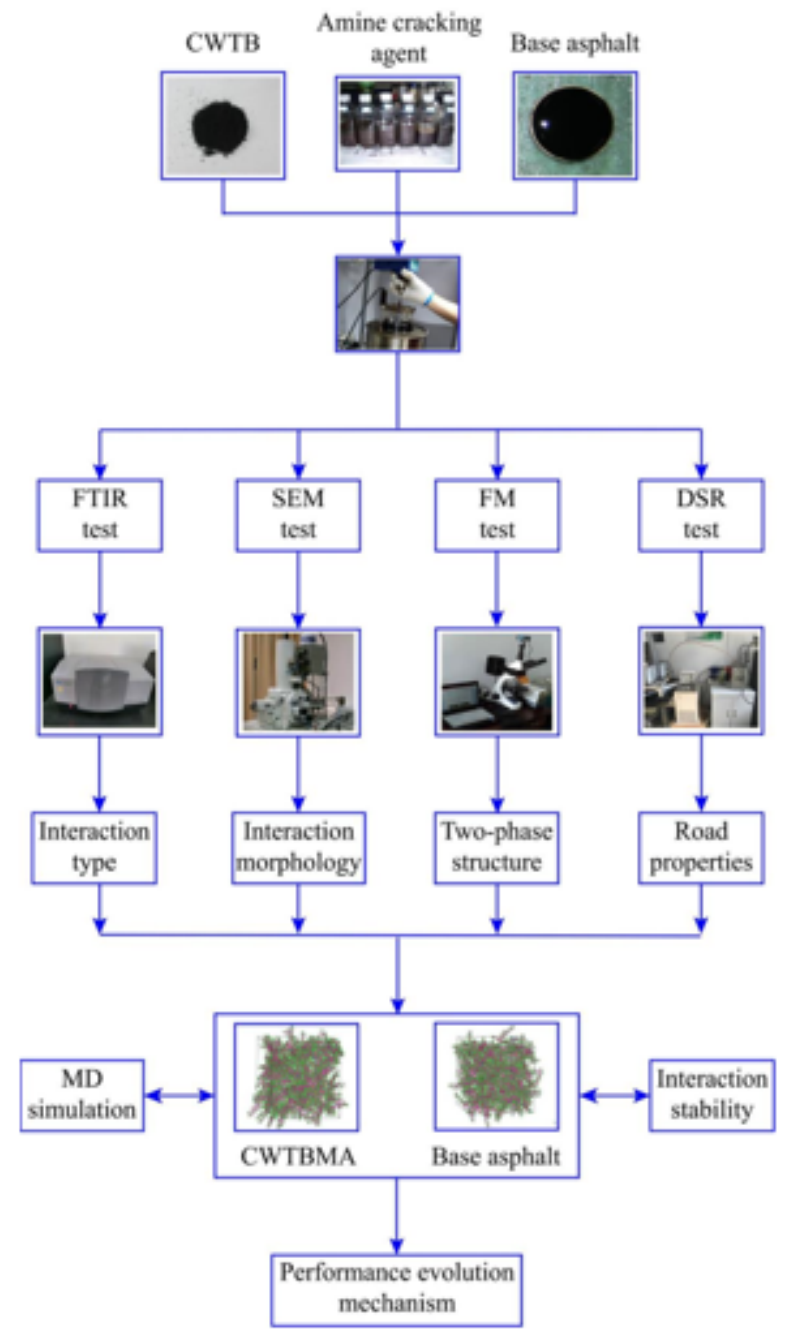

Figure 7

Technical route of this study

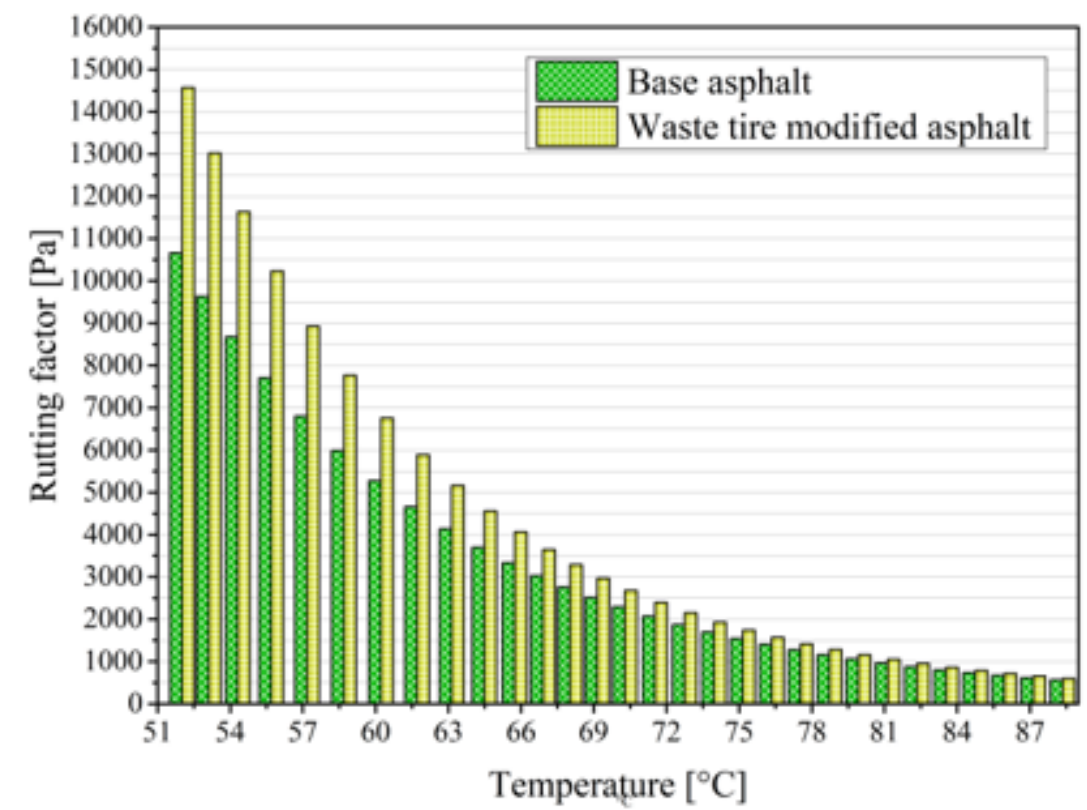


Figure 8

Rutting factor of base asphalt and CWTB modified asphalt

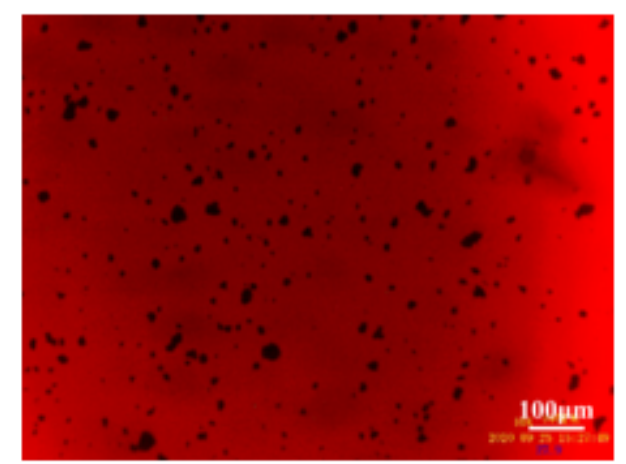

(a)

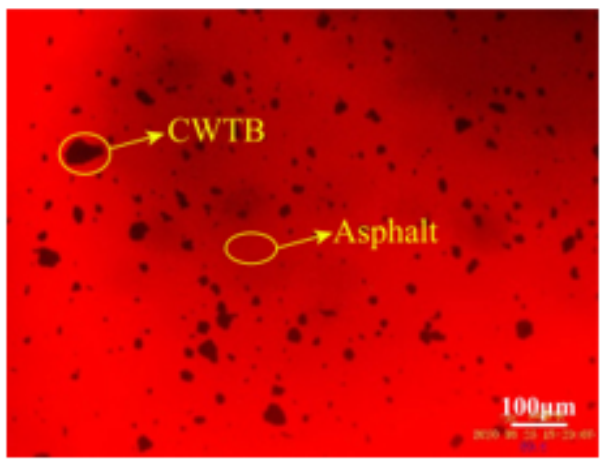

(b)

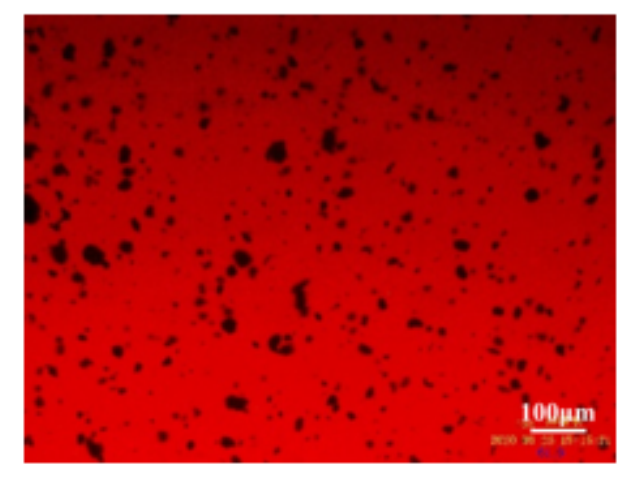

(c)

\section{Figure 9}

Two-phase structure of the development of CWTB modified asphalt at $1 \mathrm{~h}(\mathrm{a}), 2 \mathrm{~h}(\mathrm{~b})$ and $4 \mathrm{~h}(\mathrm{c})$ 


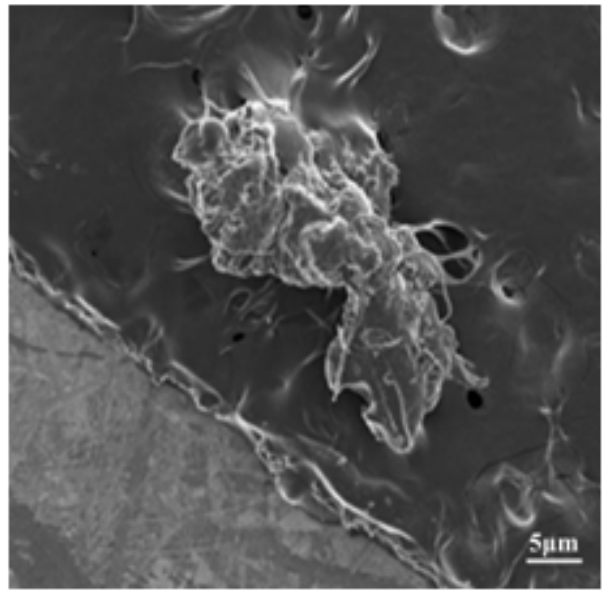

(a)

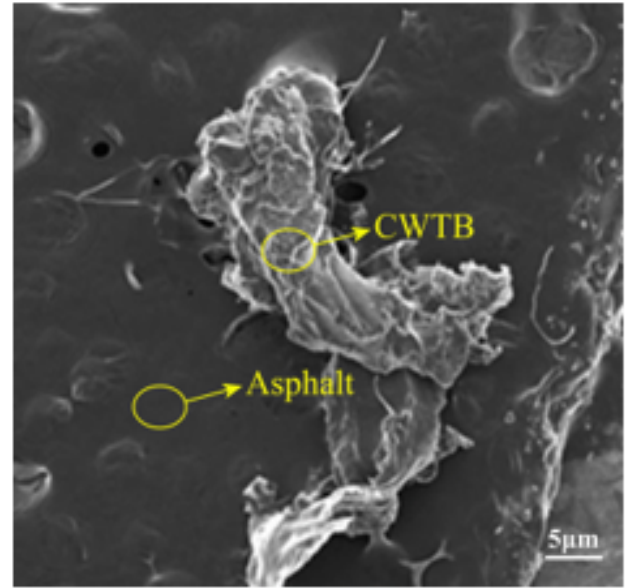

(b)

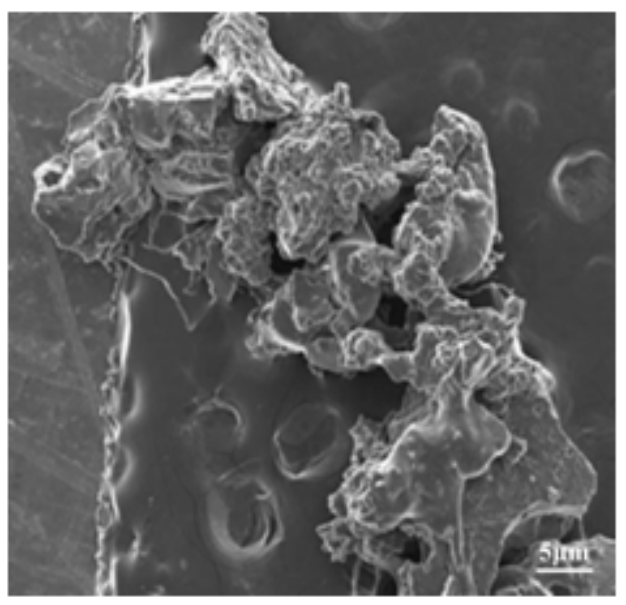

(c)

\section{Figure 10}

Microscopic morphology of the development of CWTB modified asphalt at $1 \mathrm{~h}(\mathrm{a}), 2 \mathrm{~h}(\mathrm{~b})$ and $4 \mathrm{~h}(\mathrm{c})$

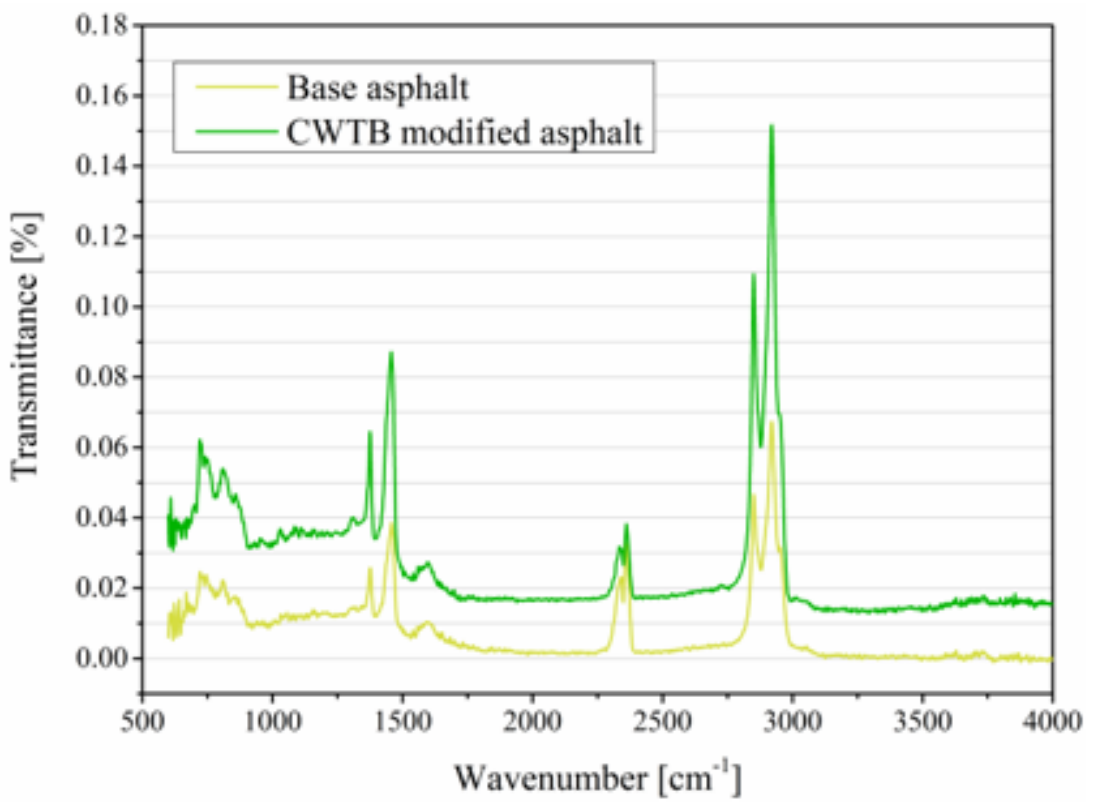


Figure 11

FTIR images of base asphalt and CWTB modified asphalt

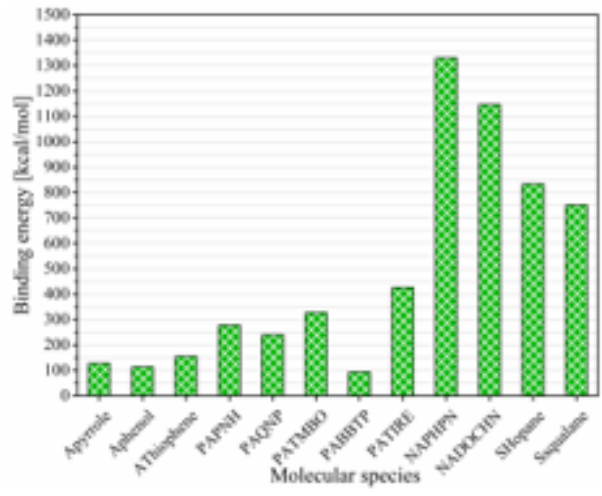

(a)

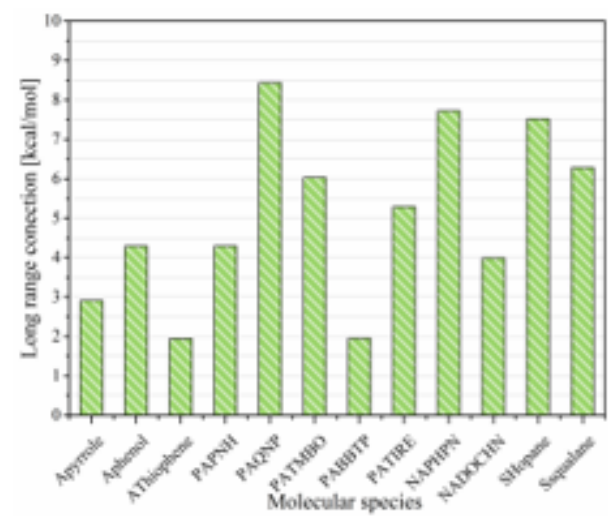

(c)

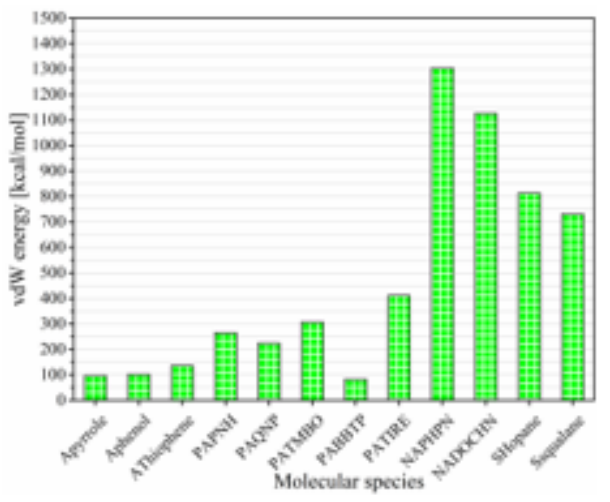

(b)

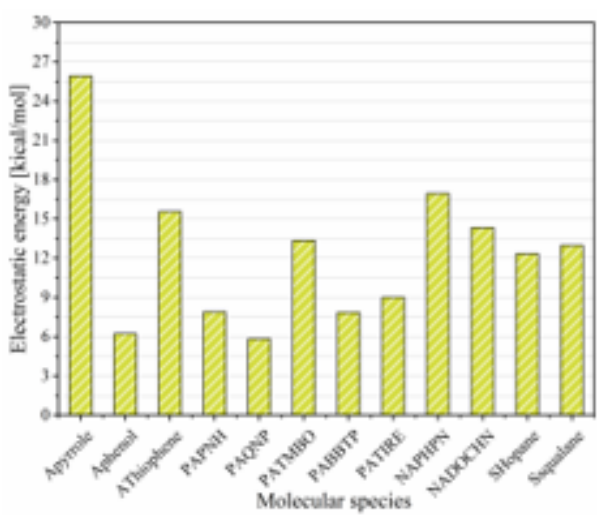

(d)

Figure 12

The binding energy between CWTB and various molecules of asphalt (a), vdW energy (b), long range correction energy (c) and electrostatic energy (d) 